\title{
Spectral domain optical coherence tomography (SD OCT) pattern of macular edema and visual acuity in recent-onset branch retinal vein occlusion (BRVO) in a tertiary hospital
}

\section{Shakya K ', Pokhrel RP², Malla OK ${ }^{3}$}

${ }^{1}$ Kiran Shakya, Assistant Professor; ${ }^{2}$ Ram Prasad Pokhrel, Professor; ${ }^{3}$ Om Krishna Malla, Professor; Department of Ophthalmology, Kathmandu Medical College Teaching Hospital, Kathmandu, Nepal

\begin{abstract}
Background: Macular edema is the common sight-threatening condition following branch retinal vein occlusion. Objective: To find spectral domain optical coherence tomography characteristics of macular involvement and visual acuity in the patients with branch retinal vein occlusion Methodology: This is a cross-sectional study; consecutive new cases of branch retinal vein occlusion diagnosed at the ophthalmology department of Kathmandu Medical College, Kathmandu were carried out from November 2016 to October 2017. All subjects were divided three groups with normotensive/controlled hypertension, stage 1 hypertension (systolic pressure $>140 \mathrm{~mm} \mathrm{Hg}$ or diastolic pressure $>90 \mathrm{~mm} \mathrm{Hg}$ ) and Stage 2 hypertension (systolic pressure $>160 \mathrm{~mm} \mathrm{Hg}$ or diastolic pressure $>100 \mathrm{~mm} \mathrm{Hg}$ ). The macular edema was evaluated by taking best corrected visual acuity and spectral domain optical coherence tomography for measurement of central foveal thickness.

Results: Forty patients with forty eyes were enrolled. Mean age was 58.5 years SD \pm 9.98 years. Stage 1 and stage 2 hypertensive groups had significantly worse best corrected visual acuity and more thickness of central foveal thickness than normotensive/controlled group $(p<0.001)$. The best corrected visual acuity and central foveal thickness were successively worse among normotensive group, stage 1 hypertensive group and stage 2 hypertensive group ( between normotensive/controlled group and stage 1 hypertensive group, $p=0.032$ and 0.002 respectively and between stage I hypertensive group and stage 2 hypertensive group, $p=0.013$ and 0.09 respectively).

Conclusion: Control of hypertension could be a main contributing measure to lower incidence of branch retinal vein occlusion and the severity of macular edema.
\end{abstract}

Key words: Central foveal thickness; Stages of hypertension; Visual acuity

\section{INTRODUCTION}

Tacular edema is the common sight-threatening 1 condition following branch retinal vein occlusion (BRVO). About 5-30\% of eyes with BRVO develop macular edema ${ }^{1,2}$. Vitreous hemorrhage develops in about $40 \%$ of eyes with BRVO over an unspecified time period and in $10 \%$ of eyes with central retinal vein occlusion (CRVO) within nine months of presentation ${ }^{3}$.

Hypertension is one of the important risk factors for BRVO and causes thickening of arteriole walls so BRVO

Address for correspondence

Dr. Kiran Shakya

Assistant professor, Department of Ophthalmology

Kathmandu Medical College Teaching Hospital

Sinamangal, Kathmandu, Nepal

E-mail: kiranshakya2001@gmail.com occurs at sites where retinal arterio- venous crossing occurs. The obstruction in venous outflow after BRVO increases intra luminal venous pressure and causes transudation of plasma and blood, resulting in macular edema and retinal edema and hemorrhages. Edema increases interstitial pressure and further compromises arterial perfusion resulting in variable amounts of capillary non-perfusion and cotton wool spots. Extensive non-perfusion is associated with ischemic BRVO and results in poor visual prognosis.

This study focused on spectral domain optical coherence tomography (SD OCT) characteristics of macular involvement and visual acuity in the patients. 


\section{METHODOLOGY}

The study was a hospital-based cross-sectional study. The subjects included in the study were those presenting to the Ophthalmology department of Kathmandu Medical College Teaching Hospital from November 2016 to October 2017. All consecutive new cases of BRVO diagnosed at the Institute with informed consent were included. Patients with diabetic mellitus, smoker, renal disease, severe dyslipidemia, intraocular inflammation or a prior history of intraocular injections, laser therapy or vitrectomy for BRVO and the patients who refused to enroll in the study were excluded from the study. Arterial blood pressure was recorded in both arms in seated position by aneroid sphygmomanometer. All subjects were divided three groups with normotensive/ prehypertensive/ controlled hypertension (defined as systolic pressure $<140 \mathrm{~mm} \mathrm{Hg}$ or diastolic pressure $<90 \mathrm{~mm} \mathrm{Hg}$ ), stage 1 hypertension (defined as systolic pressure $>140 \mathrm{~mm} \mathrm{Hg}$ or diastolic pressure $>90 \mathrm{~mm} \mathrm{Hg}$ ) and Stage 2 hypertension (defined as systolic pressure $>160 \mathrm{~mm} \mathrm{Hg}$ or diastolic pressure $>100 \mathrm{~mm} \mathrm{Hg})^{4}$. Snellen visual acuity (VA) was converted to log of the minimum angle of resolution (logMAR) for statistical analysis. The macular edema was evaluated by taking best corrected visual acuity, performing direct and indirect ophthalmoscopy with 90D and 20D and the patients were sent tertiary hospital for SD OCT (Ziess, cirrus) scan to measurement of central foveal thickness (CFT).

Ethical clearance was obtained from the Institutional Review Committee of Kathmandu Medical College, Sinamangal, Kathmandu. An informed written consent was taken from all enrolled patients. Data were entered and analyzed using statistical package for the social sciences (SPSS) version 20.0. Kruskal Wallis Test was applied for $p$ value and a $p$-value of $<0.05$ was considered statistically significant. Pearson's correlation of coefficient was applied.

\section{RESULTS}

The mean age of the patients was 58.5 years SD \pm 9.98 years (range: 42-80). Females were preponderant (72.5\%). Mean time of presentation was 16 days SD \pm 12.9 days. Seventy percent of patients gave no history of hypertension and remaining $30 \%$ gave history of hypertension under medication. Fifty percent (20) patients were having stage 2 followed by $25 \%$ (10) stage 1 and 25\% (10) were having normotensive/controlled. Superior temporal BRVO was predominant (83\%). Stage 1 and stage 2 hypertensive groups had significantly worse Best corrected visual acuity (BCVA) and more thickness of Central foveal thickness (CFT) than normotensive group $(p<0.001)$ (Table 1$)$.

Figure 1 and 2 show that the best corrected visual acuity (BCVA) and central foveal thickness (CFT) were successively worse among normotensive/prehypertensive/controlled group, stage 1 hypertensive group and stage 2 hypertensive group (between normotensive/pre-hypertensive/controlled group and stage 1 hypertensive group, $\mathrm{p}=0.032$ and 0.002 respectively and between stage 1 hypertensive group and stage 2 hypertensive group, $\mathrm{p}=0.013$ and 0.09 respectively). The systolic blood pressure had strong correlation among worsening of BCVA $(r=0.82)$ and had mild correlation among central foveal thickness $(r=$ $0.43)$, demonstrated in figures 3 and 4.

In figure 5, a fifty seven years female had systolic pressure $110 \mathrm{mmHg}$ and diastolic $70 \mathrm{mmHg}$ presented with left eye superiotemporal branch retinal vein occlusion. Her best corrected visual acuity was $6 / 6$ and central foveal thickness was $235 \mu \mathrm{m}$ showing spongy macular edema in SDOCT and in figure 6,a forty five years male had systolic pressure $170 \mathrm{mmHg}$ and diastolic $100 \mathrm{mmHg}$ presented with left eye infero temporal branch retinal vein occlusion. His best corrected visual acuity BCVA was $1 / 60$ and central foveal thickness was $621 \mu \mathrm{m}$ showing severe macular edema with subretinal fluid in SDOCT.

Table 1: Mean best corrected visual acuity (BCVA) and central foveal thickness (CFT)

\begin{tabular}{lcc}
\hline Types of hypertension & Mean BCVA(LogMAR) & Mean CFT ( $\mu$ m) \\
Normotensive $(n=10)$ & $0.40(S D \pm 0.16)$ & $285.80(S D \pm 20.48)$ \\
Stage $1(n=10)$ & $0.74(S D \pm 0.38)$ & $458.93(S D \pm 178.69)$ \\
Stage $2(n=20)$ & $1.15(S D \pm 0.42)$ & $537.65(S D \pm 148.45)$ \\
\hline
\end{tabular}


Spectral domain optical coherence tomography (SD OCT) pattern of macular edema and visual acuity in recent ...

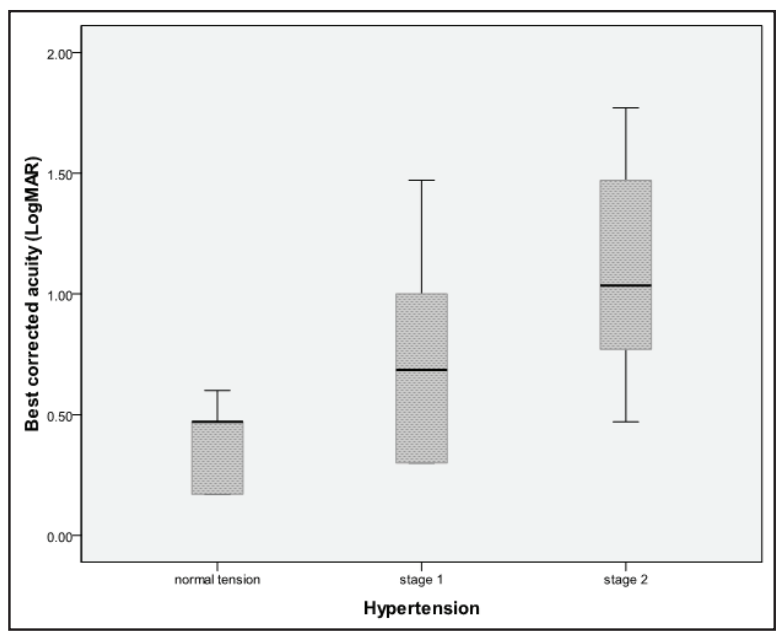

Figure 1: Medians of BCVA in different stages of hypertension among BRVO patients

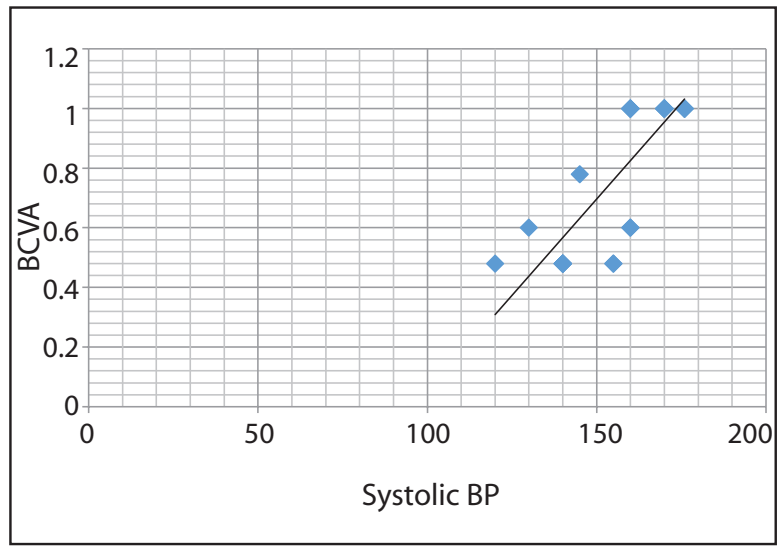

Figure 3: The strong correlation between BCVA and systolic blood pressure

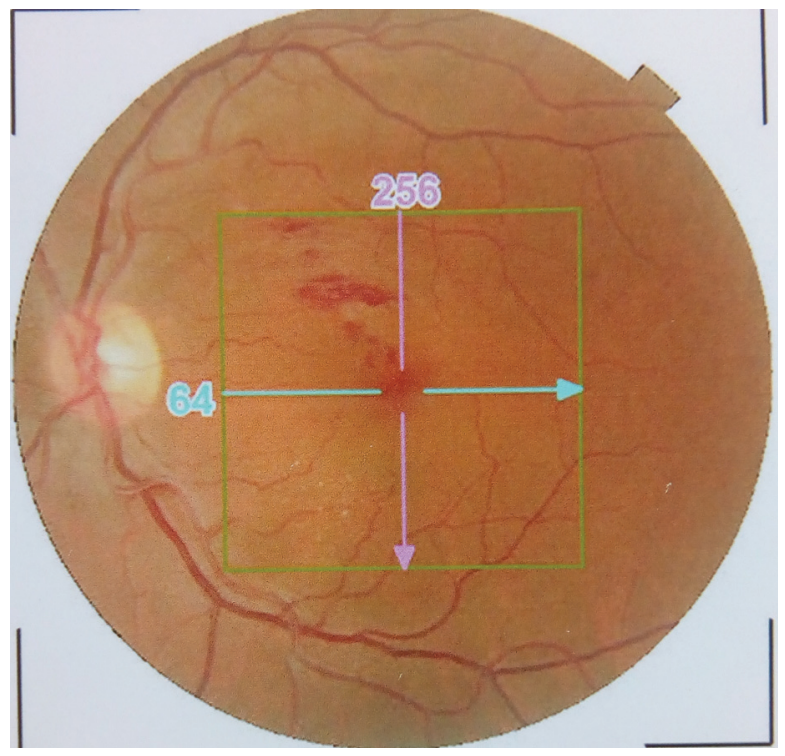

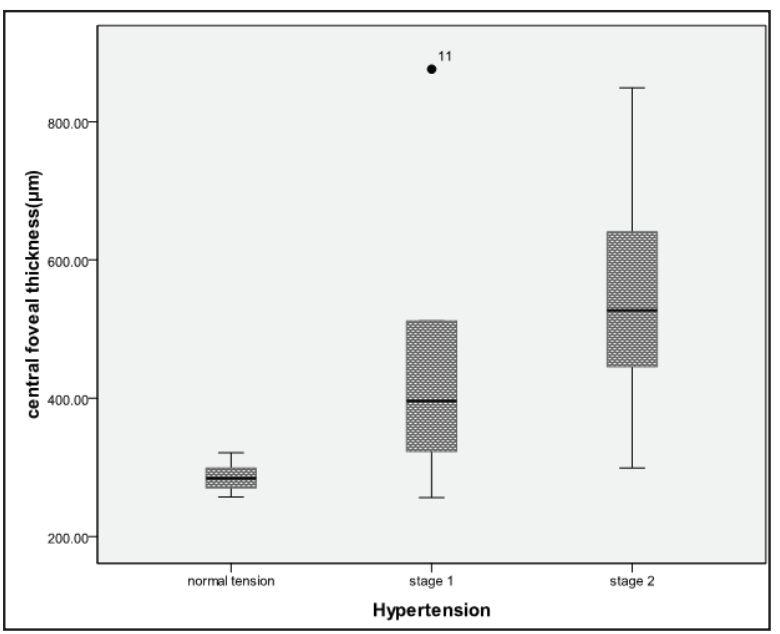

Figure 2: Medians of CFT in different stages of hypertension among BRVO patients

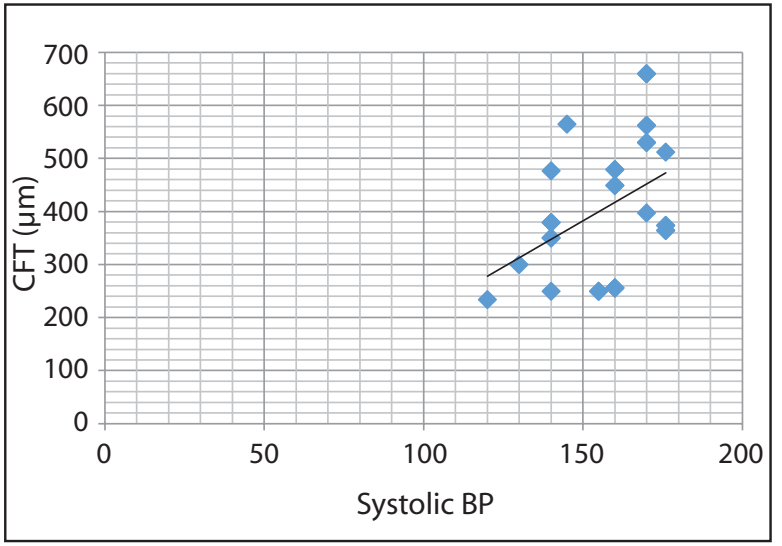

Figure 4: The mild correlation between CFT and systolic blood pressure

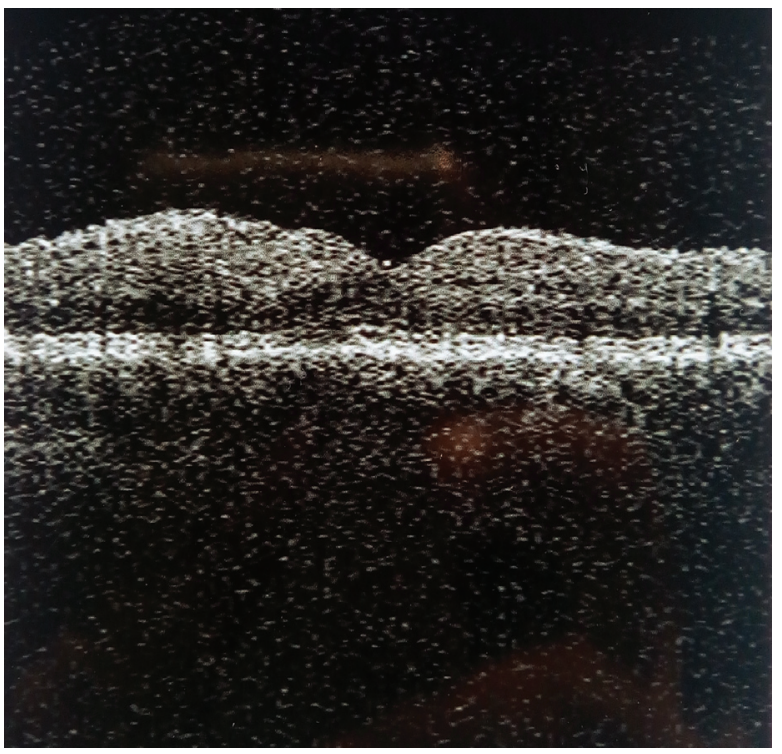

Figure 5: Left eye showing superiotemporal retinal vein occlusion (left side) and spongy macular edema (right side) in SD OCT 

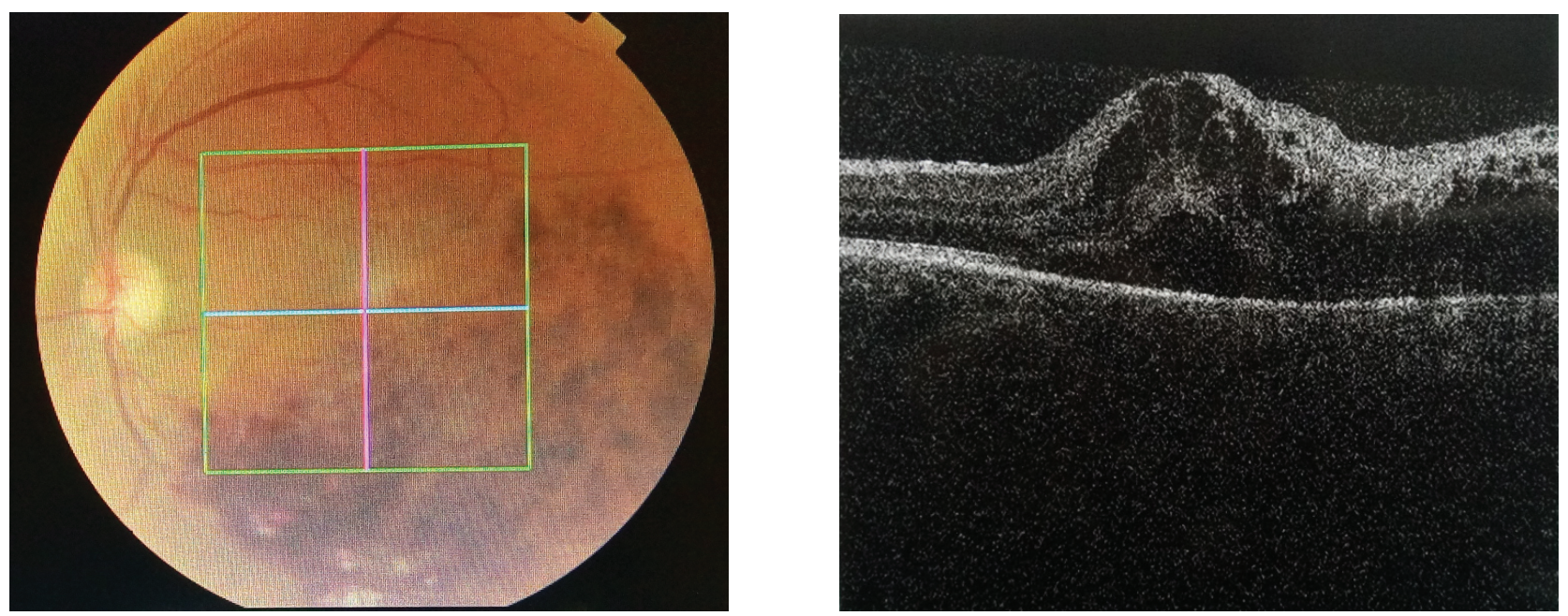

Figure 6: Left eye showing inferotemporal retinal vein occlusion (left side) with severe macular edema (right side) in SD OCT

\section{DISCUSSION}

Branch Retinal vein occlusion is the common form of retinal vascular disease with prevalence rate of $0.5-$ $2.0 \%^{5,6}$. Mean age of BRVO patients was 58.5 years $\mathrm{SD} \pm$ 9.98 years (minimum age 42 and maximum age 80 ) and our study was similar to the Beijing eye study². Laouri M et al found RVO is rarely seen in individuals younger than 50 , but may affect up to $5 \%$ of individuals over the age of $80^{5}$.Vaidya $A$ et al studied the mean age of the hypertensive population in 2006 was 40.54 ( \pm 16 ) years $(41.48 \pm 15.24$ for male and $39.83 \pm 16.53$ for female) in Kathmandu?

Fifty percentage (20) patients were having stage 2 hypertension followed by $25 \%$ (10) stage 1 hypertension and $25 \%$ (10) were having normotension/controlled. The data of published studies suggest that $48 \%$ of RVO is connected to hypertension, $20 \%$ to hyperlipidemia, and $5 \%$ to diabetes mellitus ${ }^{8}$. Systemic diseases such as hypertension, hyperlipidemia, and diabetes mellitus are very strongly associated with the development of RVO?. The study conducted in Korea shows that participants with uncontrolled hypertension had significantly more RVO than participants without hypertension ${ }^{10}$. Zhou JQ et al found that significantly higher levels of systolic and diastolic blood pressure were noted in retinal vein occlusion patients ${ }^{8}$. A hospital-based case-control study conducted in Nepal also shows a hypertension as a risk factor for RVO ${ }^{11}$. Findings from the study of Korean patients confirm the strong association of hypertension with BRVO ${ }^{12}$.

The severity of hypertension had some correlation among worsening of BCVA and macular edema. In this study the BCVA and CFT were successively worse among normotensive/controlled hypertension group, stage 1 hypertensive group and stage 2 hypertensive group. Macular edema was found in $30 \%$ of BRVO eyes ${ }^{2}$. A case series shows the blood pressure of patients was closely associated with macular edema in RVO patients and if systemic hypertension is present, it should be treated before any anti-VEGF therapy was begun ${ }^{13}$.

Female were preponderant (75\%) because the study excluded smokers who were mostly males. The prevalence of RVO was similar between men and women in all studies that reported the prevalence by gender ${ }^{3}$.

\section{CONCLUSION}

Macular edema and visual acuity of BRVO are successively worsened with severity of hypertension. So, early detection and control of hypertension could be a main contributing measure to lower the spectrum of BRVO.

\section{LIMITATIONS}

Further study should be done in large sample size to solidify the role of hypertension control in BRVO patients. 


\section{REFERENCES}

1. Mclntosh RL, Rogers SL, Lim L, Cheung N, Wang JJ, Mitchell P,et al. Natural history of central retinal vein occlusion: an evidence-based systematicreview. Ophthalmology 2010; 117(6): 1113-23. [ PubMed]

2. Zhou JQ, Xu L, Wang S, Wang YX, You QS,Tu $Y$, et al. The 10-year incidence and risk factors of retinal vein occlusion: the Beijing eye study. Ophthalmology;2013:120: 803-8.[ Full Text]

3. Rogers SL, McIntosh RL, Lim L, Mitchell P, Cheung N, Kowalski JW et al. Natural history of branch retinal vein occlusion: an evidence-based systematic review. Ophthalmology 2010; 117(6): 1094-1101.[ PubMed ]

4. Chobanian AV, Bakris GL, Black HR, Cushman WC, Green LA, Izzo JL Jr, Et al. The seventh report of the Joint national committee on prevention, detection, evaluation and treatment of high blood pressure. Hypertension. 2003;42:1206-52.[ PubMed]

5. Laouri $\mathrm{M}$, Chen $\mathrm{E}$, Looman $\mathrm{M}$ and Gallagher $\mathrm{M}$. The burden of disease of retinal vein occlusion: review of the literature. Eye 2011; 25, 981-8.[ Full Text ]

6. Klein R, Moss, Meuer SM, Klein BE. The 15-year cumulative incidence of retinal vein occlusion: the Beaver Dam Eye study. Arch Ophthalmology 2008;126:513-8.[ Full Text ]

7. Vaidya A, Pathak RP, and PandeyMR Prevalence of hypertension in Nepalese community triples in 25 years: a repeat cross-sectional study in rural Kathmandu. Indian Heart J. 2012 Mar; 64(2): 128131.[ DOI]

8. Ehlers JP and Fekrat S. Retinal vein occlusion: beyond the acute event. Survey of Ophthalmology2011; 56:281-99.[PubMed]

9. Mohamed Q, Mclntosh RL, Saw SM, and Wong TY. Interventions for central retinal vein occlusion. An evidence-based systematic review. Ophthalmology 2007;114:507-19.[PubMed]

10. Shin YU,Cho H, Kim JM, Bae K, Kang MH, Shin JP, Nam E et al. Prevalence and associated factors of retinal veinocclusion in the Korean National Health andNutritionExamination Survey, 2008-2012A cross-sectional observational study. Medicine (Baltimore). 2016 Nov;95(44):e5185.[ PubMed ]

11. Shrestha N, Byanju RN, Bhattarai B, Bajracharya K, Shrestha R.Clinico-epidemiological characteristics of central retinal vein occlusion. Nepal J Ophthalmology 2014; 6(11):39-45. [ DOI ]

12. Lee JY, Yoon YH, Kim HK, Yoon HS, Kang SW, Kim JG, et al. Baseline Characteristics and Risk Factors of Retinal Vein Occlusion: A Study by the Korean RVO Study Group.J Korean Med Sci. 2013 Jan; 28(1): 136-44.[ DOI ]

13. Kida T, Morishita S, Kakurai K, Suzuki H, Oku H, Ikeda $\mathrm{T}$. Treatment of systemic hypertension is important for improvement of macular edema associated with retinal vein occlusion. ClinicalOphthalmology. 2014; 8: 955-8.[ DOI ] 